\title{
Dalgacık Dönüşümü ve RUS Geliştirilmiş Ağaç Kullanarak Otomatik Aritmi Tespiti
}

\author{
Özgür TOMAK ${ }^{*}$, Birkut GÜLER ${ }^{2}$, Alparslan TÜFEKÇİ ${ }^{1}$, Kenan YANMAZ $^{3}$ \\ ${ }^{1}$ Giresun Üniversitesi, Elektrik ve Enerji Bölümü, Giresun, Türkiye \\ ${ }^{2}$ Giresun Üniversitesi, Makine ve Metal Teknolojileri Bölümü, Giresun, Türkiye \\ ${ }^{3}$ Giresun Üniversitesi, Elektronik ve Otomasyon Bölümü, Giresun, Türkiye
}

Geliş Tarihi: 30.03 .2018

"Sorumlu Yazar: ozgur.tomak@giresun.edu.tr Kabul Tarihi: 30.05 .2018

\section{$\ddot{\mathbf{O z}}$}

Kalp hastalıklarının çok yaygın ve ölümcül hastalıklar olduğu söylenebilir. Bu nedenle EKG incelenerek kalp rahatsızlıklarını doğru tespit etmek gerekmektedir. Bu çalışmada kişinin elektrokardiyografi (EKG) kayıtlarının otomatik incelenmesi yoluyla aritmi tespiti hedeflenmektedir. Bu süreçte dalgacık yönteminden elde edilen özellikler RUS Geliştirilmiş Ağaç yöntemiyle sınıflandırılmıştır. Kalp atışları 7 farklı sınıfa ayrılmıştır. Bu aşamada öznitelik sayısını azaltmak ve sinyal işleme sürecini hızlandırmak için RUSBoost yöntemi kullanılmıştır. Bu yöntem çok miktarda zayıf öğreniciyi bir araya getirip, bu süreçten güçlü öğrenici oluşturmak olarak bilinir. ST-Petersburg Enstitüsü Kardiyolojik Veritabanı analiz için tercih edilmiştir. Test ve eğitim doğruluğu 12 kanal EKG verilerinde bulunmuştur. Yöntem gerçek zamanlı aritmi tespiti için yeterince hızlıdır. Bütün analizler için MATLAB kullanılmıştır.

Anahtar Kelimeler: Elektrokardiyografi (EKG), Ayrık Dalgacık Dönüşümü, RUSBoost, Sınıflandırma.

\section{Automatic Arrhythmia Detection Using Wavelet Transform and RUSBoosted Trees Classification}

\begin{abstract}
It can be said that heart diseases are very common and fatal diseases. Therefore, it is necessary to determine the heart diseases correctly by examining the ECG In this study, it was aimed for detection of the arrhythmia by automatic examination of the person's electrocardiography (ECG) records. In this process, features obtained from the wavelet method was classified by RUSBoosted Trees method. The heartbeats were divided into seven different classes. RUSBoost method was used to reduce the number of features, and it speeds up the signal processing process. This method is known as bringing together a lot of weak learners and creating powerful learners from this process. ST-Petersburg Institute of Cardiological Database has been preferred for analysis. Test and training accuracy was found in 12 channel ECG data. The method was fast enough to detect real-time arrhythmia. MATLAB was used for all analyzes.
\end{abstract}

Keywords: Electrocardiogram (ECG), Discrete Wavelet Transform, RUSBoost, Classification. 


\section{Giriş}

Elektrokardiyografi (EKG) kalp hastalıklarının tespit edilmesinde yaygın olarak kullanılan biyomedikal bir sinyaldir. Aritmilerin otomatik teşhisi erken tedavi imkânı sağlamaktadır. Bu çalışmada aritmi tespiti için ayrık dalgacık dönüşümünden gelen katsayılar RUSBoost yöntemiyle azaltılmış ancak daha güçlü özellikler elde edilmiştir. Bu özellikler RUS geliştirilmiş ağaç (RUSBoosted Trees) sınıflandırma metodu kullanılarak ayrıştırılmıştır.

Bu çalışmada ST-Petersburg Enstitüsü Kardiyolojik Veritabanı (ST-Petersburg Institute of Cardiological Database) kullanılmıştır. Bu kayıtlar yaşları 18 ile 80 arasında değişen 15 kadın, 17 erkekten oluşan hasta gurubuna aittir. 12 kanall 30 dakika uzunluğunda 75 sinyal üzerinde analizler gerçekleştirilmiştir. 176177 kalp atışı harmanlanmış ve sonrasında kullanılmıştır. Her kayıt için örnekleme frekansı 257 Hz'dir. ST-Petersburg Enstitüsü Kardiyolojik Veritabanına ait örnek bir veri Şekil 1'de verilmiştir (Goldberger ve ark. 2000).

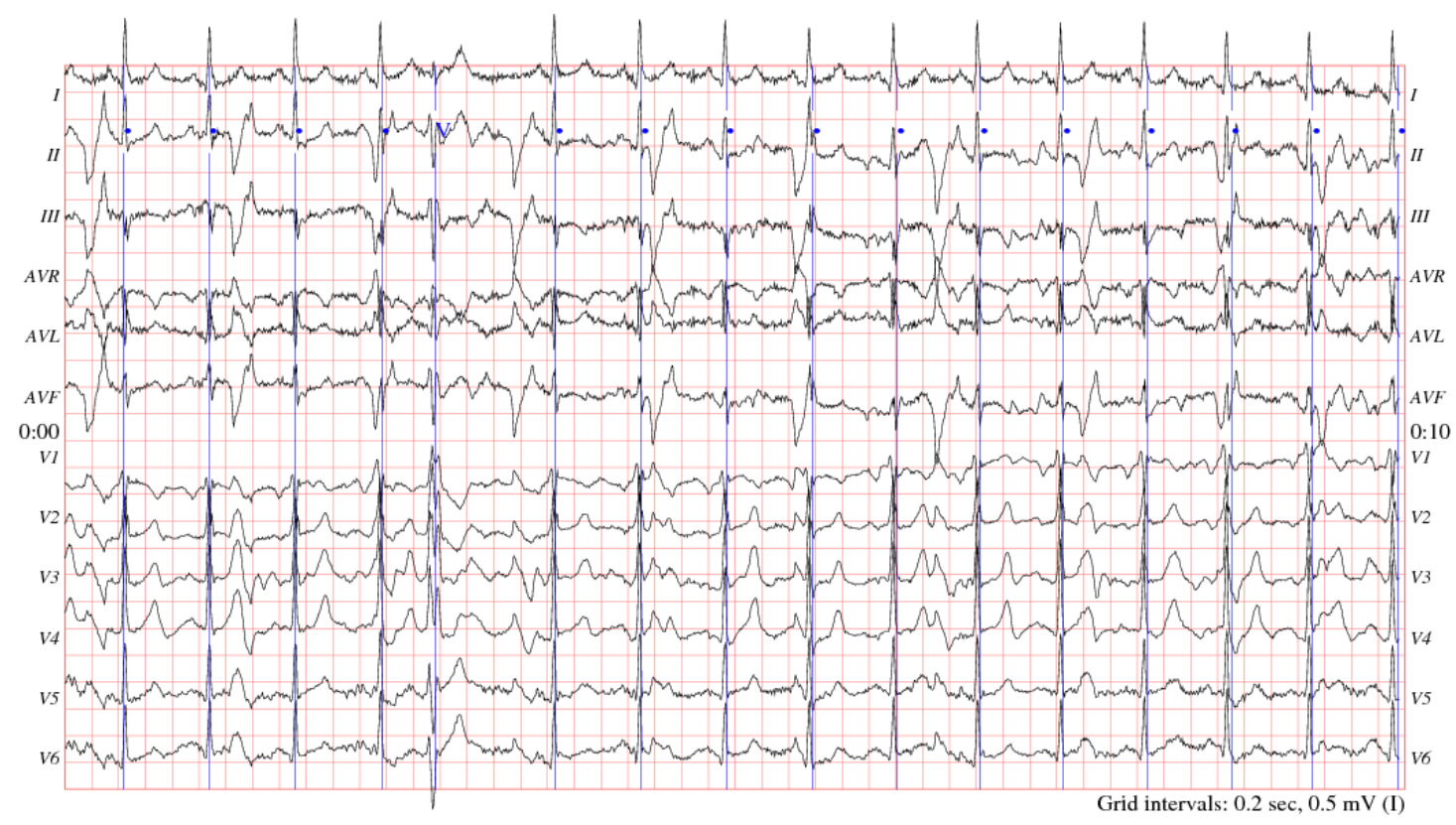

Şekil 1. ST-Petersburg Enstitüsü Kardiyolojik Veritabanına ait örnek bir veri.

EKG sinyalinde aritmi tespiti giderek önem kazanan bir çalışma konusudur. Pek çok şekilde bu konuda çalışmalar yapılmıştır. Bu çalışmaların bazılarından aşağıda bahsedilmiştir.

Dokur ve arkadaşları, çalışmalarında EKG sinyalinden Dalgacık Dönüşümü ve Fourier analizi kullanarak özellikleri belirlemişlerdir. Sinıflandırma aşamasında yapay sinir ağları ve genetik algoritmalar ve kullanılmıştır. 10 farklı aritmi tipinde sınıflandırma yapılmış, dalgacık dönüşümünde \%99.4 ve Fourier Dönüşümünde \%92.2 başarı sağlanmıştır (Dokur ve ark. 1999). Zhao ve arkadaşları, çalışmalarında her EKG sinyalinden dalgacık dönüşü ile elde ettikleri kat sayıları özellik olarak kullanmışlardır. Sonra özbağlaşımlı modelleme ile geri beslemeli ağlar oluşturup, Destek 
Vektör Makinesi kullanarak 6 farklı aritmi çeşidini \%99.68 başarıyla sınıflandırmışlardır (Zhao ve Zhang 2005). Jiang ve arkadaşları, dalgacık dönüşümünden elde ettikleri katsayılarda bağımsız bileşen analizini kullanarak elde ettikleri özellikler ile Destek Vektör Makinesi metodunu kullanarak sınıflandırma yapmışlar ve \%98.65'lik başarı elde etmişlerdir (Jiang ve ark. 2006). Prasad ve Sahambi dalgacık ailesi olarak sym6 kullanmış ve 4.seviyede ayrıştırmış ve çıkan sonuçları yapay sinir ağları ile sınıflandırılmıştır (Prasad ve Sahambi, 200). Erdoğmuş ve Pekçakar yaptıkları çalışmada dalgacık ailesi olarak db2 ve db10 kullanmış ve 4.seviyede ayrıştırmadan elde edilen katsayıları kullanarak yapay sinir ağlarında sınıflandırılmıştır (Erdoğmuş ve Pekçakar, 2009). Saini ve arkadaşları, dalgacık dönüşümü ve k En Yakın Komşular(KNN) sınıflandırıcısını kullandılar ve \% 87.5 doğruluk elde ettiler(Saini ve ark. 2015). Dewangan ve Shukla, ayrık dalgacık dönüşümü ile EKG sinyallerini incelediler(Dewangan ve ark. 2016). Yapay sinir ağları ile \% 87 doğruluk elde ettiler. Sayilgan ve arkadaşları, aritmiyi tespit için kümeleme yöntemini kullanmışlardır(Sayilgan ve ark. 2017). MITBIH Veri Tabanı'ndan alınan veriler ile analiz yapıldı ve analizlerde \% 92 doğruluğa ulaşıldı. Rad ve arkadaşları, EKG sinyallerini sınıflandırılmak için bir sistem geliştirdi ve yapay sinir ağları ve Bayesian düzenlemesi kullanarak \% 78.5 doğruluğa ulaştı ( $\operatorname{Rad}$ ve ark. 2017).

Çalışmanın takip eden kısımlarında önce özellik çıkarımı için kullanılan ayrık dalgacık dönüşümü açıklanmıştır. Sonra sınıflandırma için kullanılan RUS Geliştirilmiş Ağaç Sınıflandırması üzerinde durulmuştur. En son bölümde ise yaptığımız analiz sonuçları incelenerek yorumlanmış ve diğer çalışmalarla karşılaştırılmıştır.

\section{Kullanılan Yöntem}

Özellik olarak dalgacık yönteminden elde edilen katsayılar kullanılmıştır. EKG sinyallerinden çıkarılan bu öznitelikler sınıflandırma işlemi için RUS Geliştirilmiş Ağaç algoritmasına giriş olarak verilmiştir.

\subsection{Ayrık Dalgacık Dönüşümü}

Dikey dalgacık taban fonksiyonumuz denklem 1'de verilmiştir.

$$
\Phi_{(s, l)}(x)=2^{\frac{-s}{2}} \Phi\left(2^{-s} x-l\right)
$$

$\Phi_{(s, l)}(x)=$ Dikey dalgack taban fonksiyonu, $\quad \mathrm{l}=$ Konum indeksi, $\mathrm{s}=$ ölçek indeksi 
Ayrık dalgacıklar ailesini oluşturmak için ana fonksiyonu genişleten ve ölçekleyen değişkenler 1 ve s ‘dir. Konum indeksi 1 konumu verir ve ölçek indeksi s dalgacık genişliğini verir (Graps, 1995).

Analiz yapan dalgacık $\Phi$, veri alanının farklı çözünürlüklerde yayılması için bir denklem 2'deki gibi bir ölçeklendirme denkleminde kullanılır (Tomak, 2018).

$$
W(x)=\sum_{k=-1}^{N-2}(-1)^{k} c_{k+1} \Phi(2 x+k)
$$

$\Phi=$ Analiz yapan dalgacık,$\quad \mathrm{W}(\mathrm{x})=$ Ölçekleme fonksiyonu,

$c_{k}=$ dalgacık katsayıları

Dalgacık katsayıları denklem 3 ve 4'deki koşulları sağlamalıdır.

$$
\begin{aligned}
& \sum_{k=0}^{N-1} \quad c_{k}=2 \\
& \sum_{k=0}^{N-1} \quad c_{k} \quad c_{l}=2 \delta_{l, 0}
\end{aligned}
$$

$c_{k}$ ve $c_{l}=$ dalgac1k katsayıları, $\quad \quad l=$ konum indeksi, $\quad \delta=$ delta fonksiyonu Dalgacık türlerinin isimleri ve bazı dalgacık fonksiyonlarının çizimleri şekil 2'de verilmiştir. Sinyalin 5. Seviye $\mathrm{Db} 6$ ayrık dalgacık analizi şekil 3'de verilmiştir. Çok çözünürlüklü çift ağacı (Multiresolution dyadic tree) şekil 4'de verilmiştir. Bu çalışmada db6'nın seçilme nedeni yapısının EKG sinyaline benzemesidir. 

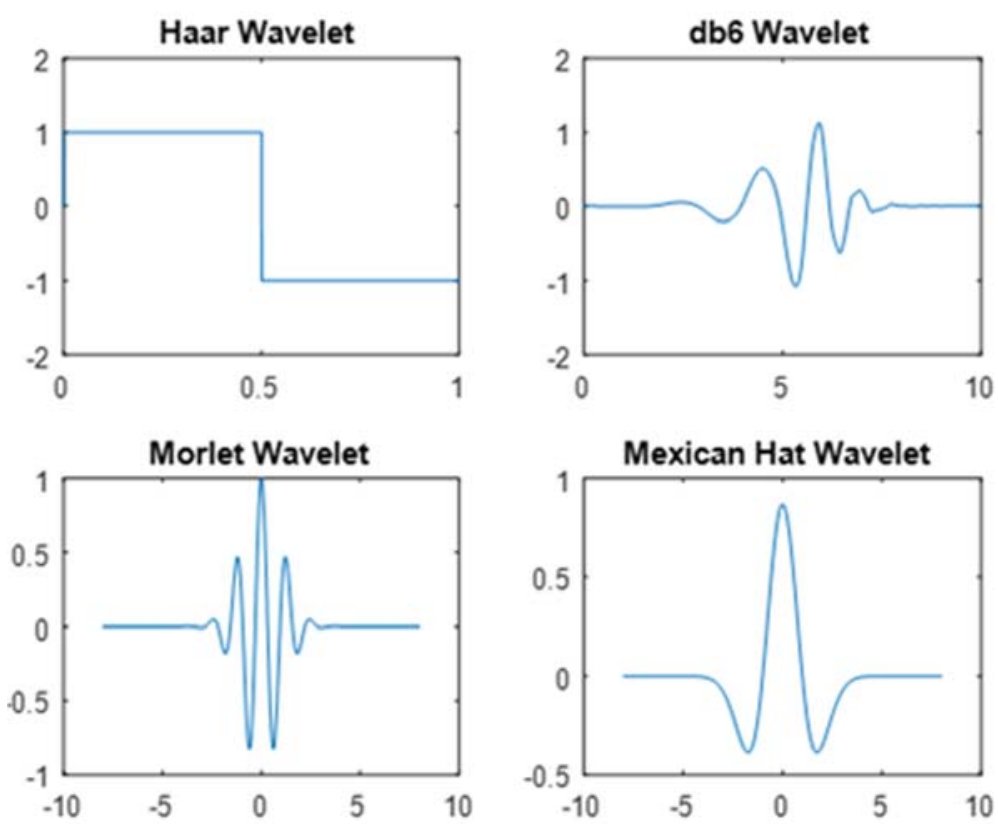

\begin{tabular}{|c|c|}
\hline Haar & haar \\
\hline Daubechies & $\mathrm{db}$ \\
\hline Symlets & sym \\
\hline Coiflets & coif \\
\hline BiorSplines & bior \\
\hline ReverseBior & rbio \\
\hline Meyer & meyr \\
\hline DMeyer & dmey \\
\hline Gaussian & gaus \\
\hline Mexican_hat & mexh \\
\hline Morlet & morl \\
\hline Complex Gaussian & cgau \\
\hline Shannon & shan \\
\hline Erequency B-Spline & fbsp \\
\hline Complex Morlet & cmor \\
\hline Fejer-Korovkin & fk \\
\hline
\end{tabular}

Şekil 2. Dalgacık türlerinin isimleri ve bazı dalgacık fonksiyonlarının çizimleri

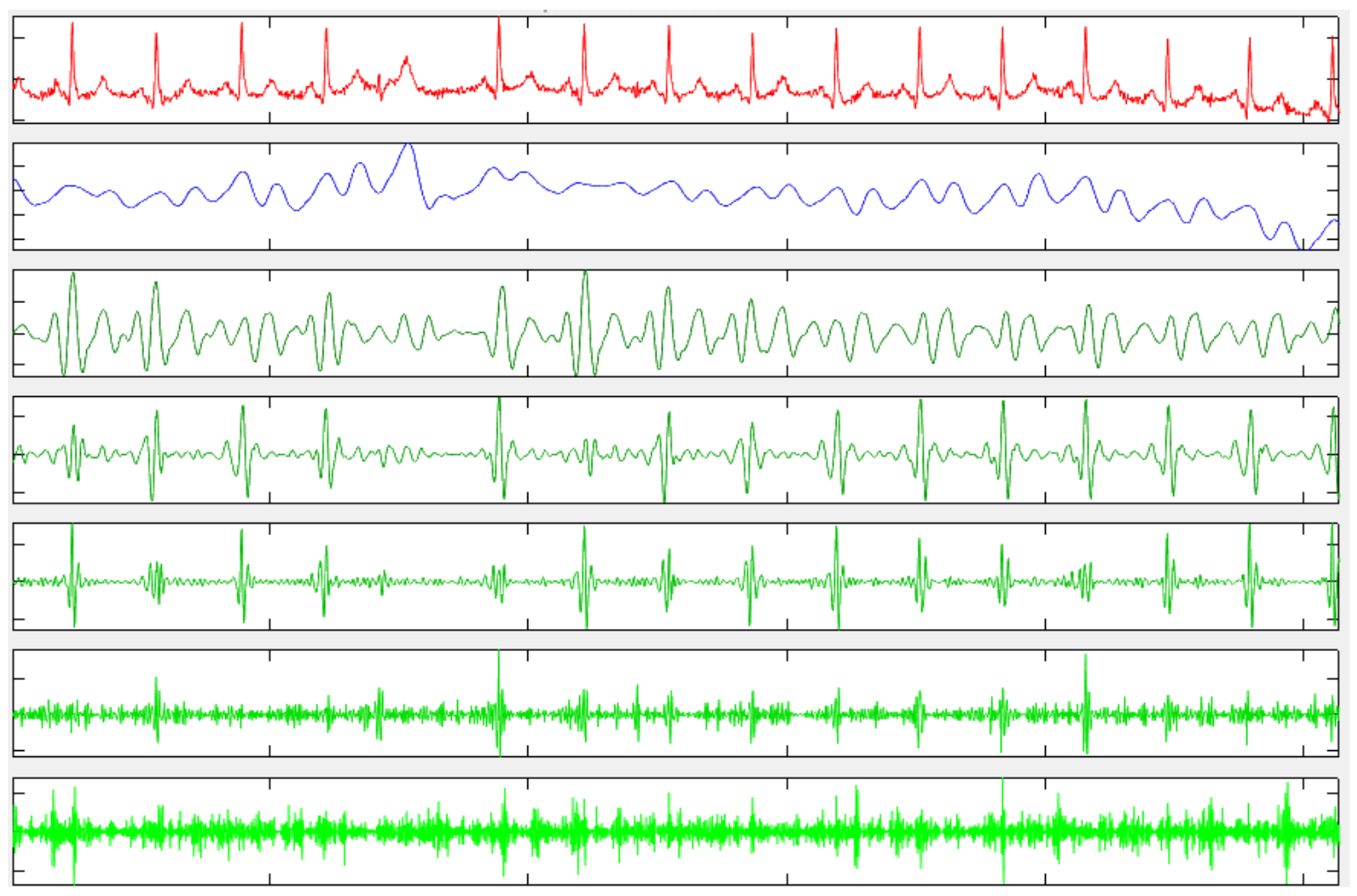

Şekil 3. 5. Seviye Db 6 ayrık dalgacık analizi. 


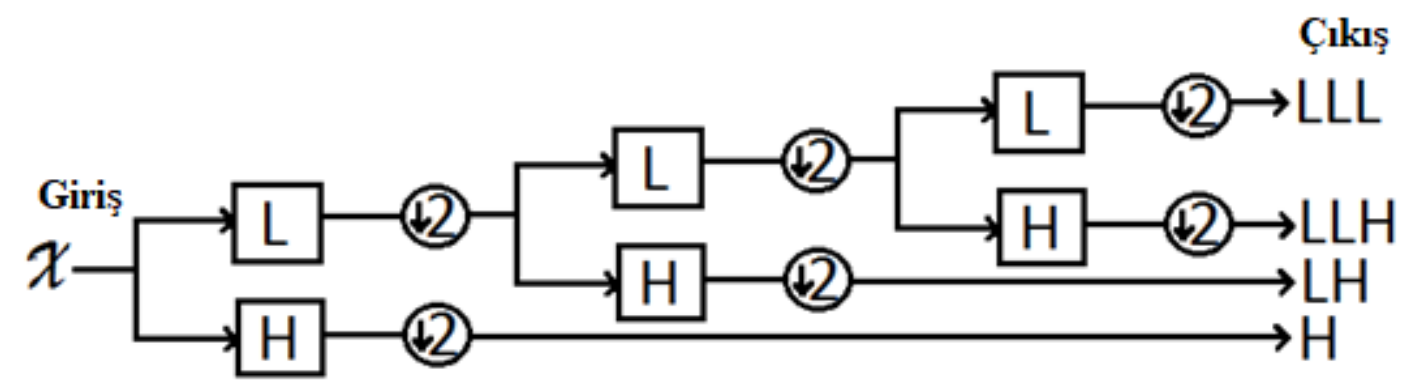

Şekil 4. Çok çözünürlüklü çift ağac1.

\subsection{RUS Geliştirilmiş Ağaç Sınıflandırması}

Sınıflandırma yöntemi olarak RUS Geliştirilmiş Ağaç (RUSBoosted Trees) tercih edilmiştir. RUS Geliştirilmiş Ağaç için grup yöntemi RUSBoost seçilmiştir Robustboost adını verdiğimiz önerilen algoritmamız, Freund tarafindan önerilen Brownboost algoritmasında bir varyasyondur. $\mathrm{Bu}$ ikisi arasındaki fark, eğitim hatasını en aza indirgemek yerine, denklem 5'de tanımlanan tolerans bazlı maliyet fonksiyonunun asgariye indirilmesidir (Freund, 2009).

$$
\mathrm{MF}=\frac{1}{N} \sum_{i=1}^{N} 1\left[\bar{m}\left(x_{i}, y_{i}\right) \leq \theta\right]
$$

Zaman değişkeni aralığı $0 \leq \mathrm{t} \leq 1$ 'dir. Toleransı $\mathrm{m}$ ile gösterilir. Hedef toleransı $\theta \geq 0$ parametresidir. Çapraz-doğrulama kullanıldı ve ayarlandı. Artan $\theta$ değeri, validasyon seti ve de eğitim setine ait olan performans değerleri arasındaki farkı azaltacaktır. $\sigma_{f}>0$ değeri, son potansiyel işlevindeki adımın eğimi ile tanımlanır. Robustboost kendini sonlandırma özelliği olan bir algoritmadır. Algoritma $t \geq 1$ olduğu zaman sona erer. Hata hedefi $\in$ eğer küçük bir değer olarak ayarlanırsa Robustboost algoritması sonlandırılmaz. Burada $\in$ değerinin doğru ayarlanması, algoritmanın uygun bir sayıda tekrarlanmasıyla sona ermesi, $\in$ 'nin minimum değerinin bulunmasıyla yapılır. $\in$ ayarı, aynı zamanda $\rho$ parametresi için değeri belirler.

RUSBoost verilerin dengesiz olduğu durumda daha faydalıdır. RUS, Random-Under-Sampling kelimelerinin ilk harflerinden oluşan bir kısaltmadır. Algoritmamız, N örneklemede eğitim için kullanılan verilerin içinde en az üye barındıran sınıfa ait üye sayısını temel olaraktan alır. Daha da fazla üye sahibi olan sınıflar ise, her sınıfa ait N gözlemleri alır ve örneklendirilir. Farlı bir şekilde anlatırsak, eğer K adet sınıflar var ise, o zaman, zayıf öğreniciler için, toplulukta, RUSBoost, K sınıf var ise $\mathrm{N}$ gözlemleriyle verilere ait bir alt küme alınır. AdaBoostM2'deki yolu topluluk oluşturmada ve değerlendirmede kullanabilir. AdaBoost.M2 zayıf baz sınıflandırıcıları ile çok-sınıf problemleri 
için tasarlanmış bir destek algoritmasıdır. Algoritma, eğitim hatası üzerinde gevşek olan sınırı en aza indirecek şekilde tasarlandı.

Öğrenme tipi karar ağacıdır. Karar ağaçları, hızlı sınıflandırma için en iyi seçeneklerden biridir ve ayrıca yorumlanması kolaydır. Öğrenen sayısı 30, öğrenme oranı 0.1, maksimum bölünme sayısı 20 seçildi.

Kalp atışları 7 farklı sınıfa ayrılmıştır. Bunlar normal atım, erken ventriküler kasılma, atriyal erken atım, düğüm kaçış vuruşu, supraventriküler erken atım, sağ dal bloğu, tanımlanamayan atım şeklinde sınıflandırılabilir. ST-Petersburg Enstitüsü Kardiyolojik Veritabanından aldığımız 176177 kalp atışını harmanlanarak, yarısı eğitim ve diğer yarısı test aşamasında kullanıldı. Eğitimde 10 kat katlama (10 fold) yaklaşımı kullanılmıştır. Sinyalin 12 kanal için db6 ile 5. seviye ayrıştırmasından elde edilen katsayılar RUSBoost yöntemiyle daha güçlü özniteliklere çevrildi ve RUS Geliştirilmiş Ağaç metoduyla MATLAB kullanılarak sınıflandırıldı. Bu sınıflandırma süreci için MATLAB'da bulunan fonksiyonlar kullanıldı. Elde edilen sonuçlar Tablo 1'de verilmiştir. Kullandığımız veriye ait 12 kanala ait ilk 10 saniyelik veri Şekil 5'de verilmiştir.

Eğitim

Doğruluk

\begin{tabular}{ccc}
\hline Kanal 1 & 94.1 & 93.9 \\
\hline Kanal 2 & 94.5 & 93.6 \\
\hline Kanal 3 & 93.7 & 92.5 \\
\hline Kanal 4 & 95.1 & 94.2 \\
\hline Kanal 5 & 93.9 & 93.1 \\
\hline Kanal 6 & 93.5 & 92.6 \\
\hline Kanal 7 & 92.3 & 91.6 \\
\hline Kanal 8 & 94.1 & 93.4 \\
\hline Kanal 9 & 94.7 & 93.9 \\
\hline Kanal 10 & 93.1 & 93.0 \\
\hline Kanal 11 & 94.4 & 93.6 \\
\hline Kanal 12 & 93.4 & 93.1 \\
\hline
\end{tabular}

Tablo 1. Eğitim ve test doğruluk sonuçları 

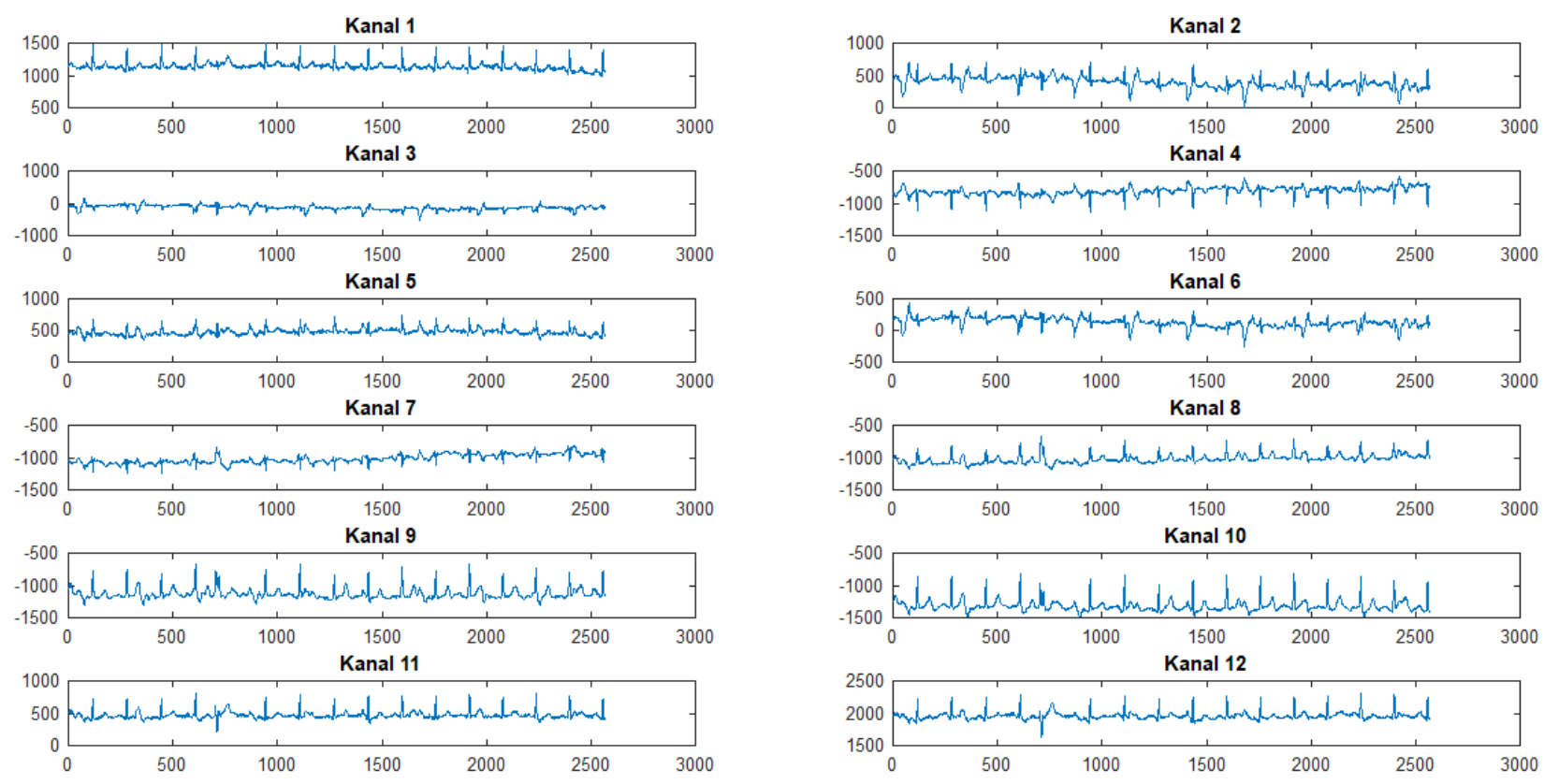

Şekil 5. 12 kanala ait ilk 10 saniyelik veri.

\section{Sonuçlar ve Öneriler}

Sınıflandırmada aşamasında kullandığımız veri ST-Petersburg Enstitüsü Kardiyolojik Veritabanından geldi ve veri miktarı 176177 kalp atışından oluşmaktadır Ayrık Dalgacık Yöntemiyle elde edilen katsayılardan RUSBoost yöntemiyle daha güçlü öznitelikler elde edilmiş ve RUS Geliştirilmiş Ağaç (RUSBoosted Trees) metoduyla sınıflandırılmıştır. Benzer çalışmalarda tek kanal ile çalışılırken yaptığımız çalışmada 12 kanalda analiz yaptık. Benzer çalışmaların en yüksek başarı seviyeleri ile kendi en yüksek başarı seviyemizin karşılaştırılması Tablo 2'de verilmiştir. Elde edilen sonuçlar her kanal için başarılıdır ve yöntem gerçek zamanlı aritmi tespiti için yeterince hızlıdır yani her kalp atışına ait hesaplamalar yine bir kalp atışı süresi içinde yapılabilmektedir. Veri miktarında azalma sinyalin gönderilmesini ve işlenmesini kolaylaştırmaktadır.

\section{Doğruluk}

\begin{tabular}{cc}
\hline Dokur ve arkadaşları & 99.4 \\
\hline Zhao ve arkadaşları & 99.68 \\
\hline Jiang ve arkadaşları & 98.65 \\
\hline Saini ve arkadaşları & 87.5 \\
\hline Dewangan ve Shukla & 87 \\
\hline Sayilgan ve arkadaşları & 92 \\
\hline Rad ve arkadaşları & 78.5 \\
\hline Yöntemimiz & 94.2 \\
\hline
\end{tabular}

Tablo 2. Benzer çalışmaların doğruluk sonuçları 


\section{Kaynaklar}

Dewangan NK, Shukla SP. ECG arrhythmia classification using discrete wavelet transform and artificial neural network. In: Recent Trends in Electronics, Information \& Communication Technology (RTEICT), IEEE International Conference on; 20-21 May 2016; Bangalore, India: IEEE. pp. 1892-1896.

Dokur, Z., Olmez, T., and Yazgan, E. (1999). ECG waveform classification using the neural network and wavelet transform. In [Engineering in Medicine and Biology, 1999. 21st Annual Conference and the 1999 Annual Fall Meetring of the Biomedical Engineering Society] BMES/EMBS Conference, 1999. Proceedings of the First Joint (Vol. 1, pp. 273-vol). IEEE.

Erdoğmuş, P., and Pekçakar, A. (2009). Dalgacık dönüşümü ile ekg sinyallerinin özellik çıkarımı ve yapay sinir ağları ile sınıflandırılması. Uluslararası İleri Teknolojiler Sempozyumu, 13-15.

Freund, Y. (2009). A more robust boosting algorithm. arXiv preprint arXiv:0905.2138.

Goldberger, A. L., Amaral, L. A., Glass, L., Hausdorff, J. M., Ivanov, P. C., Mark, R. G., ... and Stanley, H. E. (2000). Physiobank, physiotoolkit, and physionet. Circulation, 101(23), e215-e220.

Graps, A., An Introduction to Wavelets, IEEE Computational Science and Engineering, vol. 2, num. 2. IEEE Computer Society, Loas Alamitos-CA, USA, 1995.

Jiang, X., Zhang, L., Zhao, Q., and Albayrak, S. (2006, November). ECG arrhythmias recognition system based on independent component analysis feature extraction. In TENCON 2006. 2006 IEEE Region 10 Conference (pp. 1-4). IEEE.

Prasad, G. K., and Sahambi, J. S. (2003, October). Classification of ECG arrhythmias using multi-resolution analysis and neural networks. In TENCON 2003. Conference on Convergent Technologies for the AsiaPacific Region (Vol. 1, pp. 227-231). IEEE.

Rad AB, Eftestol T, Engan K, Irusta U, Kvaloy JT, Kramer-Johansen J, Wik L, Katsaggelos AK. ECG-based Classification of Resuscitation Cardiac Rhythms for Retrospective Data Analysis. IEEE T Bio-Med Eng 2017; 64.10: 2411-2418.

Saini R, Bindal N, Bansal P. Classification of heart diseases from ECG signals using wavelet transform and kNN classifier. In: Computing, Communication \& Automation (ICCCA), 2015 International Conference on; 15-16 May 2015; Noida, India: IEEE. pp. 1208-1215.

Sayilgan E, Cura ÖK, İşler Y. Use of clustering algorithms and extreme learning machine in determining arrhythmia types. In Signal Processing and Communications Applications Conference (SIU) 2017 25th; 15-18 May 2017; Antalya, Turkey: IEEE. pp. 1-4.

Tomak Özgür, (2018). Dalgacık Teorisi Kullanarak Güç Kalitesi Ve Geçici Durumların Analizi. Yüksek Lisans Tezi, Giresun Üniversitesi, Fen Bilimleri Enstitüsü, Giresun.

Zhao, Q., \& Zhang, L. (2005, October). ECG feature extraction and classification using wavelet transform and support vector machines. In Neural Networks and Brain, 2005. ICNN\&B'05. International Conference on (Vol. 2, pp. 1089-1092). IEEE. 